\title{
Abnormal Trend Detection of Sequence-Disordered Data Using EWMA Method ${ }^{*}$
}

\author{
Jr-Min Fan ${ }^{1}$, Ruey-Shan Guo ${ }^{2}$, Shi-Chung Chang ${ }^{3}$, Jian-Huei Lee ${ }^{4}$ \\ ${ }^{1,3}$ Department of Electrical Engineering, National Taiwan University \\ ${ }^{2}$ Department of Industrial and Business Administration, National Taiwan University \\ ${ }^{4}$ Taiwan Semiconductor Manufacturing Corporation
}

\begin{abstract}
In this paper, we focus on the design issues of applying the exponentially weighted moving average (EWMA) chart to end-of-line electrical test data. Since the sequence of end-of-line test data is not the same as the sequence in each process step, an abnormal trend in any of the process steps is more difficult to detect based on end-of-line test data than based on single step process data (if available). Our approach uses EWMA chart because the moving average is able to smooth out the sequence-disordered effect and the weighting factor allows us to choose an effective moving average size. The correlation among weighting factor, detection speed, and sequence-disordered effect is studied. Fab data is used to verify the effectiveness of EWMA chart for detecting process shifts if we appropriately choose the weighting factor based on the derived correlation.
\end{abstract}

\section{Introduction}

In an advanced wafer fab, both in-line data and endof-line data are collected and monitored to improve fab yield rate [1]. In-line data includes wafer, process or equipment measurements taken at specific process steps. Process control techniques such as response surface methods, Taguchi method, and statistical process control (SPC) charts use these in-line measurements to monitor and control process performance during both design and manufacturing. End-of-line data mostly refers to the wafer electrical test data after completing the whole fabrication process of a wafer. It provides important information regarding process integration status, which is usually difficult to assess through in-line data only. When measured end-of-line data shows out-of-spec values or abnormal trends such as drifts and shifts, integration engineers must find out the root causes as quickly as possible and feed such information back to process engineers to prevent further yield crisis.

\footnotetext{
* This work was supported in part by the National Science Council of the Republic of China under Grant NSC85-2212-E-002-074 and Taiwan Semiconductor Manufacturing Corporation under Contract 84-S-119.
}

Process control techniques such as acceptance sampling and SPC charts use these end-of-line measurements for specification checking and abnormal trend detection respectively.

In this paper, we focus on the abnormal trend detection of end-of-line data using SPC charts. SPC chart techniques, however, cannot be applied to end-ofline data without caution. Since the sequence of end-ofline data is not the same as the sequence in each process step, our empirical data characterization indicates that an abnormal trend in any of the process steps becomes ambiguous for detection based on end-of-line data. Our approach adopts exponentially weighted moving average (EWMA) chart [2][3], where moving average smooths out the "sequence-disordered" data and weighting factor allows us to choose an effective moving average size so that the underlying trend can be popped out.

In the next section, one industry practice on end-ofline quality control and trend detection is reviewed. In section 3, we describe the generation process of end-ofline data and compare the sequence of end-of-line data with that of in-line data. Design issues of applying EWMA chart to end-of-line data are then addressed in section 4 . In section 5, real fab data is used to verify the effectiveness of applying EWMA chart to detect process shifts. Conclusions are made in section 6.

\section{End-of-line Quality Control Practice}

From the quality control point of view, quality should be built in as early as possible, first in the design stage, then in the manufacturing stage and finally in the end-ofline quality check. Although being the last resort, endof-line quality control still provides opportunities to enhance quality for the following reasons:

r1. End-of-line data provides important information regarding process integration status, which is usually difficult to assess through in-line data only. A slight change of several in-line process settings may cause a significant effect on the end product, 
although in-line measurements are all monitored as under control.

r2. In-line data might be missing or not available. The collection of in-line data is usually time consuming and intrusive.

r3. There are usually abundant end-of-line electrical test measurements stored in the engineering data base. Most of such end-of-line data is not well exploited.

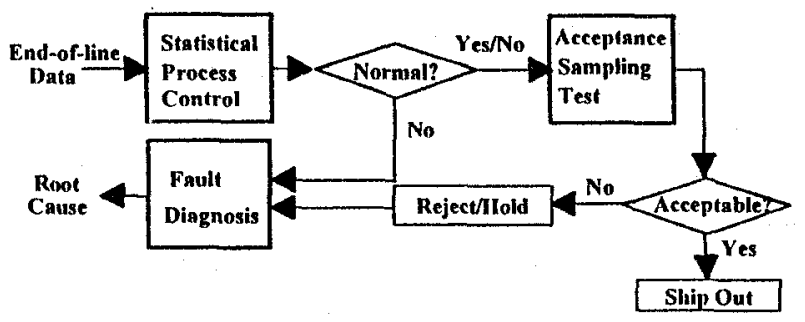

Figure 1 An end-of-line quality control framework

In order to effectively exploit the end-of-line data, an end-of-line quality control system has been proposed in a wafer fab [7]. Figure 1 illustrates the modules of the system which consists of statistical process control, acceptance sampling test and fault diagnosis. Here, abnormal trend detection is accomplished in the SPC module, specification is clecked in the acceptance sampling test module and root cause finding is accomplished in the fault diagnosis module. In this paper, we focus on the SPC module.

A current fab practice of end-of-line SPC adopts a heuristic, bi-weekly SPC review, to monitor the "sequence-disordered" end-of-line data. Under this method, the process capability metric Cpk [5] of key endof-line data is monitored every two weeks. If any of the Cpk values is less than the corresponding threshold values specified by engineers, a problem might have occurred and the corresponding control charts must be reviewed to see if there is any significant trend. The

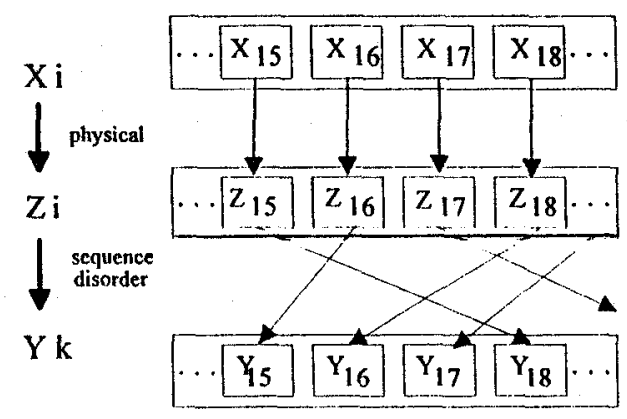

Figure 2 End-of-line data generation process philosophy of this method is to reduce the sequencedisordered effect by plotting the data in a two-week time period so that the trend pattern could be identified more easily. Several problems arise with the use of this method:

ql. The threshold value of each end-of-line item is empirical.

q2. The trend patterns are identified by engineers subjectively.

q3. Two-week time period for trend detection is too long

\section{Sequence-disordered Effect}

To overcome the deficiency of the current practice, we first characterize the sequence-disordered effect by analyzing the end-of-line data generation process. Capturing the problem features, we then propose an EWMA control chart technique to enhance the detection resolution and speed.

Let $\left\{X_{i}\right\}$ be a random sequence representing wafer lot averages of a measurement item taken at process step $P$, and $\left\{Y_{k}\right\}$ be a random sequence representing wafer lot averages of a physically related measurement item (to $X$ ) taken at the end of line. For example, $\left\{X_{i}\right\}$ may correspond to an in-line measurement such as dopant concentration and deposited layer thickness of lot $i$ at step $P$, and $\left\{Y_{k}\right\}$ may correspond to an electrical test measurement such as threshold voltage and saturation current. Based on the physical laws, $\left\{Y_{k}\right\}$ can be expressed as a function of $\left\{X_{i}\right\}$.

In general, affected by different process flows and dispatching policies, the cycle time from process step $P$ to the end-of-line step is not constant among lots. As a result, the lot with sequence label $i$ at step $P$ very likely has a different lot sequence label $k$ at the end-of-line step. Define the sequence-disordered grade $R$ of

$Z_{i}$
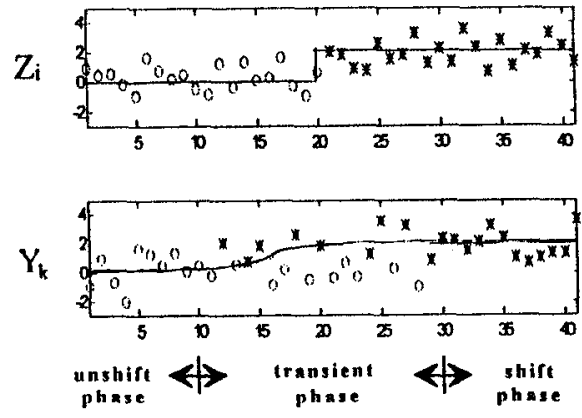

Figure 3 Sequence-disordered effect 
the lot from step $P$ to the end of line as $k-i$. Further define $Z_{i}=Y_{k}$. Then $\left\{Z_{i}\right\}$ is a sequence that reorders $\left\{Y_{k}\right\}$ according to lot sequence at step $P$. Figure 2 illustrates the end-of-line data generation process.

To monitor the trend in process step $P$, we can exploit the sequences $\left\{X_{i}\right\},\left\{Z_{i}\right\}$ or $\left\{Y_{k}\right\}$. Usually, $\left\{X_{i}\right\}$ may not be available as stated in $r 2$. There is also a limitation to track back $\left\{Z_{i}\right\}$ from the historical data base. Because there are too many process steps need to be monitored. The required computer memory and processing time would be too much if we trace all process steps. Therefor, we exploit the end-of-line sequence $Y_{k}$ to monitor the process trend.

An abnormal trend in any of the process steps would be more difficult to detect based the observed $Y_{k}$ because of the sequence-disordered effect, which is illustrated in Figure 3 by an example of a shift in a process step's setting. The plot of $Y_{k}$ can be divided into three phases: inshifted phase, transient phase and shifted phase. As can be seen, the shift is not so obvious based on $Y_{k}$. Theoretically, the sequencedisordered effect increases the variance of $Y_{k}$ during the transient phase, which results in a larger fluctuation in the transient phase as compared to that of a nondisordered sequence $\left\{Z_{i}\right\}$. As a result, the trend pattern is destroyed and the detection becomes more difficult. Our approach is to estimate the true mean of $\left\{Z_{i}\right\}$ based on the exponentially weighted moving average of $\left(Y_{k}\right)$ as described in section 4 .

\section{Exponentially Weighted Moving Average}

\subsection{Moving Average}

The fluctuation of observation data caused by sequence-disordered effect can be viewed as a type of noise. Let $R^{*}$ be the range of the sequence-disordered grade $R$. If $R^{*}$ is increased, the variance of the noise caused by sequence-disordered effect is increased. Usually, the moving average (MA) approach can be adopted to smooth out the noise of observation data and estimate the process mean [4]. The moving average with size $m$ of a sequence $\left(Y_{k}\right)$ is defined by

$$
B_{k}=\frac{1}{m} \sum_{n=0}^{m-1} Y_{k-n} .
$$

The larger the variance of noise, the larger the MA size needed to smooth it out.

Traditional process control charts such as Shewhart chart and cumulative sum (CUSUM) chart can be viewed as special cases of $\mathrm{M} \Lambda$ chart. However, they are insufficient in the trend detection of sequence-disordered data. The Shewhart chart corresponds to an MA with $m=1$. It signals an out of control based on the last data point or identifies a trend based on the last few data points with the help of run rules. When applied to sequence-disordered data, it is too sensitive to the last few data points and results in too many false alarms. On the contrary, the CUSUM chart corresponds to MA with $m=k$ for the $k$ th data point and is insensitive in identifying a small drift by weighting all the historical data points. Although it does not generate too much false alarms, the detection speed might be too low because a large subgroup size is usually used. The compromise between the two approaches is the pure MA method or exponentially weighted moving average (EWMA) method. In a pure $M A$ method, equal weight is assigned to the data points while in an EWMA method, higher weights are assigned to the later data points. The weighting schemes among the four approaches are illustrated in Figures 4 and 5 [2] respectively.

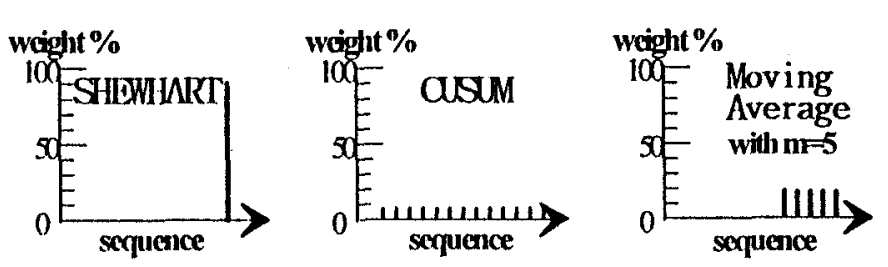

Figure 4 Data weighting for the Shewhart, CUSUM and pure moving average chart
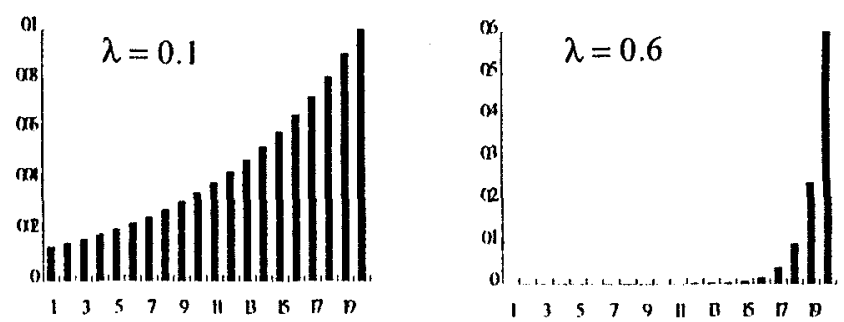

Figure 5 The decaying behavior of EWMA weighting coefficient 


\subsection{EWMA Properties}

Assume that the random variable $Y_{k}$ defined in Section 3 follows a normal distribution with mean $\mu_{0}$ and variance $\sigma^{2}$. The successive values generated by applying EWMA to $\left\{Y_{k}\right\}$ are described by

$$
\begin{aligned}
A_{k} & =\lambda Y_{k}+(1-\lambda) A_{k-1} \\
& =\sum_{n=0}^{k} W_{k-n} Y_{k-n},
\end{aligned}
$$

where $W_{k-n}=\lambda(1-\lambda)^{n}, \quad A_{0}=\mu_{0}, \quad$ and $0<\lambda \leq 1 . \quad$ The control limits of $\left\{A_{k}\right\}$ are derived as [2]

$$
\mu_{0} \pm h \sqrt{\lambda /(2-\lambda)} \sigma \text {, }
$$

where $h$ is an adjustable parameter usually specified as 3 for a false alarm rate of $0.3 \%$. It is clear that $A_{k}$ is a moving average of $\left\{Y_{k}, Y_{k-1}, \ldots, \quad Y_{1}, A_{0}\right\}$ with exponentially decreasing weighting coefficients.

There are two features of the EWMA estimator. The first one is that it tends to emphasize on utilizing the most recently collected data. The level of emphasis on recent data can be controlled by selecting the weighting factor $\lambda$. When a very small value of $\lambda$ is selected, $A_{k}$ at any time $k$ carries with it a great amount of inertia from the past, hence it is relatively robust to short lived changes in the process such as noise. If a fast response to a process shift is desired, a relatively large $\lambda$ should be used. Figure 5 shows the decaying behavior for some commonly used values of $\lambda$ [5].

The second feature of EWMA is that we can use it to resemble the pure MA by carefully selecting $\lambda$. For the same observation data $\left\{Y_{k}\right\}$, let the successive values generated by EWMA with a weighting factor $\lambda$ and by pure MA with a size $m$ be $\left\{A_{k}\right\}$ and $\left\{B_{k}\right\}$ respectively. If $\left\{A_{k}\right\}$ and $\left\{B_{k}\right\}$ has the same mean and variance, then an EWMA with a weighting factor $\lambda$ is equivalent to a pure MA with a size $m$. By this definition, the relationship between the weighting factor $\lambda$ and the effective moving average size $m$ can be easily derived (appendix B) as

$$
m=\frac{2-\lambda}{\lambda} .
$$

Based on the two features, the appropriate choice of the weighting factor makes EWMA adaptive to the philosophy of Shewhart, CUSUM and pure MA charts.

\subsection{Application}

To detect a process change sensitively, a large weighting factor is needed. However, it will result in a small effective moving average size and can not smooth out the fluctuation caused by sequence-disordered effect and other noises. Here, we use the concept of run length $(R L)$ to choose the appropriate weighting factor trading off between the detection speed and false alarm rate. The run length is a random variable characterizing the number of observations from the time a level shift occurs until the shift is clearly indicated by a control chart. The appropriate weighting factor of an EWMA control chart is the one which results in a large run length $R L 1$ when the process is under control so that the false alarm rate is low and a short run length $R L 2$ when a shift has occurred so that the detection speed is high. Usually, we use the average value $A R L 1$ of $R L 1$ and the percentage point $N_{\alpha}$ of $R L 2$ as metrics to evaluate the performance of an EWMA chart [6]. The metric ARL1 represents the average run length when process is under control. It reflects the average number of observations between false alarms of an EWMA chart subject to nature noise and should be as large as possible. The metric $N_{\alpha}$ represents a threshold value that the run length will be less than it with a probability of $\alpha$ when there is a shift in the process. It reflects the detection speed of an EWMA chart subject to a shift of the mean. The value of $N_{\alpha}$ is a positive integer determined from the following two probability inequalities:

$$
\begin{aligned}
& \operatorname{Pr}\left(R L 2<N_{\alpha}-1\right) \leq \alpha, \text { and } \\
& \operatorname{Pr}\left(R L 2<N_{\alpha}\right)>\alpha .
\end{aligned}
$$

where $0<\alpha<1$. Figure 6 shows the relationship among sequence-disordered range $R^{*}$, weighting factor $\lambda$, $A R L 1$ and $N_{0.95}$ of $R L 2$ based on the run length distribution function discussed in appendix $\mathrm{C}$.

We can see that as $\lambda$ is small, $m$ becomes large. Then EWMA is robust to noise and able to smooth out the sequence-disordered data but the detection speed becomes slow. On the contrary, if $\lambda$ is increased, the detection speed of EWMA is increased but not robust to noise, and the detection speed has a limit, e.g. around $\lambda=0.1$ for $R^{*}=20$, when the effective MA size $m$ becomes too small to smooth out the sequencedisordered data. As the sequence-disordered range $R^{*}$ is increased, a smaller $\lambda$ is preferred because a larger $m$ 

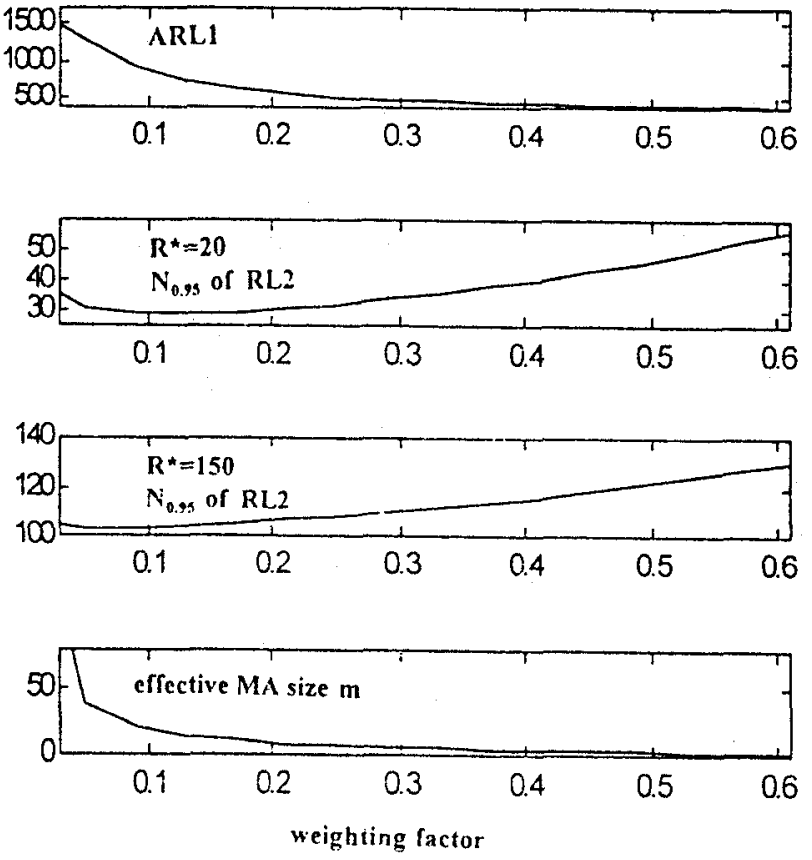

Figure 6 The relationship among sequence-disordered range $R^{*}$, weighting factor $\lambda$, and $N_{0.95}$ of RL2

is needed to smooth out the fluctuation data caused by the stronger sequence-disordered effect

\section{Fab Data Validation}

Consider a real fab case where there was a shift in the temperature of one furnace tube used for source/drain annealing. The shift amount of in-line measurements is so slight to be statistically significant. However, the nature of source/drain mean has been changed. This phenomena caused the end-of-line electrical measurements RS_P ${ }^{*}$ (the resistance of source/drain) to drop significantly. Figure 6 shows the related end-ofline data processed by using CUSUM, pure MA with $n=39$ and EWMA schemes, where all data has been normalized. Open circles represent end-of-line data $\left\{Y_{k}\right\}$ and solid lines represent various MA values. All $M A$ lines clearly indicate a trend pattern but the end-ofline raw data, which implies an earlier detection of the abnormal trends by using MA schemes.

Based on the lot sequence defined at wafer start step and end-of-line step, the disordered range $R^{*}$ between the sequences $Z_{i}$ and $r_{k}$ is less than 170 . We apply the analysis in section 4 and obtain that an appropriate value
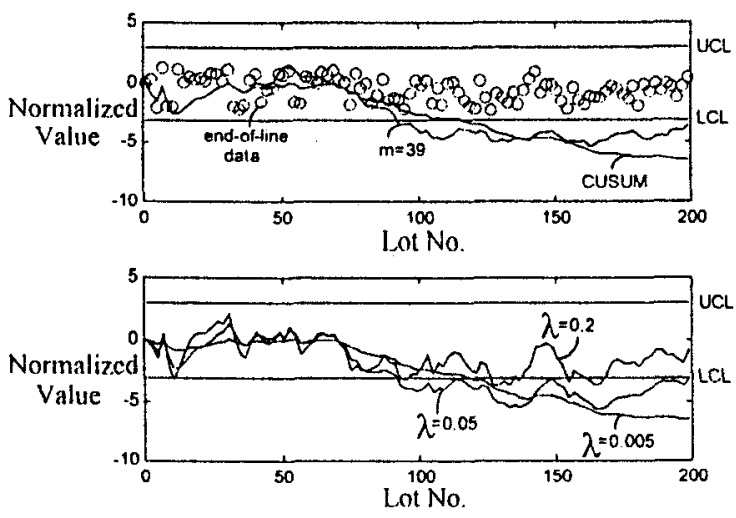

Fig. 7 Fab data validation for a shift case

for the EWMA parameter $\lambda$ is about 0.05 when $R^{\circ}=150$, which corresponds to an effective MA size of 39 . In Figure 7 , we can see that both lines corresponding to $\lambda=0.05$ and $m=39$ perform equally well and are more sensitive to process change than other lines. As $\lambda$ increases to 0.2 , the resolution of the corresponding line deteriorates because the effective MA size is too small to smooth out the sequence-disordered data. When $\lambda=1$, EWMA values are the same as $\left\{Y_{k}\right\}$, which resembles the Shewhart chart and shows no significant trend. On the other hand, as $\lambda$ decreases to 0.005 , the corresponding line is very close to the CUSUM line which is not sensitive to the process change.

All the EWMA properties derived in section 4 are verified in this case study. Moreover, we find that the pure MA performs as well as EWMA if we choose the MA size based on Eq.(4). But the EWMA method is preferred for implementation because the current EWMA value can be easily updated by the current end-of-line data and the previous EWMA value as shown in Eq.(2).

\section{Conclusions}

In this paper, the importance of end-of-line quality control is discussed. Among the many challenging issues, we describe our work on abnormal trend detection of end-of-line data focusing on resolving the sequencedisordered effect. The EWMA chart is adopted because the moving average is able to smooth out the sequencedisordered data and the weighting factor allows us to choose an effective MA size. Our analysis of the correlation among weighting factor, detcction speed, and the sequence-disordered effect provides rules of applying the EWMA chart technique. Trial implementation using fab data justifies effectiveness of EWMA chart for detecting process shifts based on the rules we derived. 


\section{Appendix}

\section{(A) Probability distribution of $Y_{k}$}

Let the mean and variance of $Z_{i}$ be $\mu_{0}$ and $\sigma^{2}$ respectively if $i<i^{*}$. When $i=i^{*}$, an abnormal event occurs and changes the mean of $Z_{i}$ from $\mu_{0}$ to $\mu_{1}$. Given the probability distribution function of the sequence-disordered grade $R$, we can infer the mean of $Y_{k}$ as $\mu_{0}$ with probability

$$
a=\operatorname{Pr}\left(k-R<i^{*}\right) \text {. }
$$

or $\mu_{1}$ with probability $(1-a)$. So, the probability density function of $Y_{k}$ can be expressed as

where

$$
f_{Y_{k}}=a f_{Y_{k} \mid \text { unshift }}+(1-a) f_{Y_{k} \mid \text { shitt }} \text {, }
$$

$$
\begin{aligned}
& f_{Y_{k} \text { lunshitt }} \sim N\left(\mu_{0}, \sigma^{2}\right), \text { and } \\
& f_{Y_{k} \text { Ishift }} \sim N\left(\mu_{1}, \sigma^{2}\right) .
\end{aligned}
$$

The mean and variance of $Y_{k}$ are then

$$
\begin{aligned}
E\left\{Y_{k}\right\} & =a \mu_{0}+(1-a) \mu_{1}, \\
\operatorname{Var}\left\{Y_{k}\right\} & =a(1-a)\left(\mu_{1}-\mu_{0}\right)^{2}+\sigma^{2} .
\end{aligned}
$$

During the transient phase, i.e. $0<a<1$, the variance will be greater than the original value $\sigma^{2}$.

\section{(B) Effective moving average size}

The mean and variance of $A_{k}\left(B_{k}\right)$ is $\mu_{0}\left(\mu_{0}\right)$ and $\frac{\lambda \sigma^{2}}{2-\lambda}\left(\frac{\sigma^{2}}{m}\right)$ respectively. So the relationship between $\lambda$ and its effective MA size $m$ is $m=\frac{2-\lambda}{\lambda}$.

\section{(C) Run Length Distribution}

The distribution of $R L 1$ is derived in [6]. For the distribution of $R L 2$, we use a procedure similar to that described in [3]. First, divide the interval between the upper and lower control limits of $A_{k}$ into $2 n+1$ subintervals of equally width $2 \delta . A_{k}$ is said to be in state $(j)$ if $S^{j}-\delta<A_{k}<S^{j}+\delta$ for $j=-n,-n+1, \ldots, n$ where $S^{j}$ represents the midpoint of the $j$ th subinterval. Based on Eq.(2), the probability that $A_{k}$ in state (i) given $A_{k-1}$ in state $(j)$ is

$$
t_{k, k-1}^{i, j} \approx \frac{2 \delta}{\lambda} f_{Y_{k}}\left(\frac{S^{i}-(1-\lambda) S^{j}}{\lambda}\right),
$$

where $f_{Y_{k}}$ is derived as Eq.(8). Let $T_{k, k-1}$ be a $(2 n+1) \times(2 n+1)$ transition probability matrix with element $t_{k, k-1}^{i, j}$ in the $i$ th raw and $j$ th column. Given a $(2 n \times 1)$ initial probability vector [3]

$$
P_{0}=\left(p^{-n}, \ldots, p^{-1}, p^{0}, p^{+1}, \ldots, p^{+n}\right)^{T},
$$

where $p^{j}$ represents the probability that $A_{0}$ in $\operatorname{state}(j)$, then

$$
\operatorname{Pr}(R L 2>k)=1\left(T_{k, k-1}\right)\left(T_{k-1, k-2}\right) \cdots\left(T_{1,0}\right) P_{0},
$$

where 1 is the raw vector $=[11 \ldots 1]$.

\section{References}

[1] R.C. Leachman, "The competitive semiconductor survey: second report on results of the main phase," U.C. Berkeley, 1994.

[2] J.S. Hunter, "The exponentially weighted moving average," Journal of Quality Technology, vol.18, no.4, 1986, pp. 203-210.

[3] J.M. Lucus and M.S. Saccucci, "Exponentially weighted moving average control schemes: properties and enhancements," Technometrics, vol.32, no.1, 1990, pp. 1-12

[4] P.J. Brockwell and Richard A. Davis, Time Series : Theory and Methods, New York : Springer-Verlag, 1991.

[5] R.E. Devor, T. Chang and J.W. Sutherland, Statistical Quality Design and Control: Contemporary Concepts and Method, New York : Macmillan, 1992.

[6] F.F. Gan, "Computing the Percentage Points of the Run Length Distribution of an Exponentially Weighted Moving Average Control Chart," Journal of Quality Technology, vol.23, no.4, 1991, pp. 359. 365 .

[7] J.H. Lee and J.S. Wei, Personal Communications. 